\title{
Cyclic rifaximin therapy effectively prevents the recurrence of symptoms after exacerbation of symptomatic uncomplicated diverticular disease: a retrospective study
}

\author{
Anna M. Pietrzak ${ }^{1,2}$, Adam Dziki ${ }^{3}$, Tomasz Banasiewicz ${ }^{4}$, Jarosław Reguła ${ }^{1,2}$ \\ ${ }^{1}$ Department of Oncological Gastroenterology, Maria Sklodowska-Curie Memorial Cancer Centre, Institute of Oncology, \\ Warsaw, Poland \\ ${ }^{2}$ Department of Gastroenterology, Hepatology and Clinical Oncology, Centre of Postgraduate Medical Education, Warsaw, Poland \\ ${ }^{3}$ Department of General and Colorectal Surgery, USK-WAM University Teaching Hospital, Lodz, Poland \\ ${ }^{4}$ General and Endocrine Surgery and Gastroenterological Oncology Department, Poznan University of Medical Sciences, \\ Poznan, Poland
}

Gastroenterology Rev 2019; 14 (1): 69-78

DOI: https://doi.org/10.5114/pg.2019.83428

Key words: symptomatic uncomplicated diverticular disease, diverticulitis, rifaximin, eubiosis.

Address for correspondence: Anna M. Pietrzak MD, PhD, Department of Oncological Gastroenterology, Maria Sklodowska-Curie Memorial Cancer Centre, Institute of Oncology, 5 Roentgena St, 02-781 Warsaw, Poland, e-mail: anpietrzak@gmail.com

\begin{abstract}
Introduction: Symptomatic uncomplicated diverticular disease (SUDD) is the most common manifestation of diverticulosis. Data concerning the optimal treatment after SUDD exacerbation are inconsistent.

Aim: To assess the effectiveness and necessity of cyclic rifaximin treatment for recurrent SUDD symptoms and for preventing exacerbations in patients who responded to the initial treatment.

Material and methods: A retrospective observational study was performed in 2017. Physicians responded to a survey on patients with recurrent SUDD during the observation period, who were cyclically treated with rifaximin $400 \mathrm{mg}$ b.i.d. for 7 days per month. The patients' SUDD history, diagnostic methods, treatment, and results were evaluated.

Results: In total 294 patients were included in this study (67\% women, median age: 65 years (26-87)). The mean duration of diverticular disease (DD) was 4.5 years (1-20), and $88 \%$ had at least one repeated episode of SUDD exacerbation before rifaximin. A total of 267 patients were treated with rifaximin. Changes in the severity of pain, abdominal tenderness, diarrhoea, constipation, and bloating were assessed every 2 months. After 6 months of rifaximin treatment there was a statistically significant reduction in the total severity score (median from 1.8 (max. 3 points) to $0.2 ; p<0.0001$; sum from 9.37 (max. 18 points) to $1.35 ; p<0.0001$ ) and an improvement in individual symptom score.

Conclusions: Cyclical rifaximin is effective in treating exacerbation of SUDD. This regimen leads to a gradual cessation of symptoms over a 6-month period. In patients who responded to the initial treatment, cyclic rifaximin therapy is needed to maintain remission.
\end{abstract}

\section{Introduction}

Diverticula, the most common finding in the digestive tract, are often detected during colonoscopy or radiological examination. Their prevalence increases with age and can be found in more than half of people aged 60 years or older [1-3]. Although most individuals are asymptomatic, a subgroup of patients develops mainly mild to moderate symptoms, such as abdominal pain, bloating, and alerted bowel habits, without evidence of inflammation in laboratory tests or radiological examinations. This condition is defined as symptomatic uncomplicated diverticular disease (SUDD) and is the most common manifestation of diverticular disease (DD), affecting up to $20-25 \%$ of patients with diverticulosis [4]. Currently, acute diverticulitis (AD) is diagnosed less frequently, in 1 to $8.5 \%$ of patients with diverticulosis or DD $[5,6]$. However, in absolute terms, AD (with or without bleeding) is the most common cause of hos- 
pitalisation related to gastrointestinal diseases in the developed world [7]. The pathogenesis of symptomatic DD is complex, multifactorial, and still under discussion. New insights have emerged in recent years regarding irreversible changes in the intestinal microbiota, which appear to be crucial for the occurrence and persistence of symptoms [8-10]. This observation was confirmed by a recent study by Barbara et al., who found qualitative and quantitative changes in microbiota composition and diversity between patients without diverticula and those with SUDD [10]. This is particularly important because it offers the potential of a targeted causal treatment for this highly common digestive disease, but it comes with a high risk of serious complications, including death [1].

The precise criteria for the various manifestations of DD were established in the second decade of the $20^{\text {th }}$ century. Because of the differences in definitions, earlier studies cannot be directly compared. Nevertheless, the main principles of treatment are outlined by many national guidelines [11]. Several treatments are currently recommended, but their effectiveness is still under discussion. Considering the aetiology of DD, the proposed drug used in the treatment of this disease is the poorly absorbable eubiotic rifaximin (a positive modulator of the intestinal ecosystem, which restores eubiosis via various mechanisms) [12]. Rifaximin is also known for its direct and indirect anti-inflammatory mechanisms via inhibition of transcription factors and cytokines through the pregnane $X$ receptor and reduction of bacterial virulence, adhesion, and translocation. Data concerning the optimal treatment (in terms of treatment duration and dose) after an exacerbation of SUDD is still unsatisfactory $[6,11,13]$. According to the guidelines, treatment with rifaximin should last for at least 12 monthly cycles [11]; however, we do not know for sure what the minimum and maximum number of treatment cycles should be in the different patient groups (first episode, relapse, post-diverticulitis) to maintain the benefits of this regimen. To date, two studies confirming the effectiveness and progressive gain after three treatment cycles in patients with the first episode of SUDD have been published $[14,15]$.

Therefore, we conducted a retrospective observational survey study assessing the effectiveness of prolonged cyclic rifaximin treatment in a group of patients with multiple recurrences of symptoms that are not considered AD.

\section{Methods according to STROBE [16] Study design and setting}

This was a retrospective observational survey study conducted in gastroenterological outpatient clinics throughout Poland over a 6-month period in 2017.
The aim of the study was to investigate whether cyclic (7 days per month) use of rifaximin (Xifaxan, Alfasigma S.p.A.) for at least 6 months is beneficial in achieving and maintaining remission of symptoms in patients with previous episodes of SUDD, who responded to an initial 7-day induction treatment.

All patient data were anonymised, and the study was performed in accordance with the ethical standards of the institutional and national research committees. Due to the retrospective epidemiologic survey character of the study there was no need to obtain informed consent.

Through our screeners, we invited gastroenterologists, surgeons, and internal medicine specialists experienced in managing patients with DD, all of whom worked in an outpatient setting, to participate in the survey. A total of 100 doctors from 61 cities covering all geographical regions in Poland agreed to participate in the study. The surveys were collected between March and August 2017, during which time physicians had to select from their database consecutive patients with SUDD, who responded to an initial 7 days of treatment and were then cyclically treated with rifaximin and regularly visited their clinic for up to 6 months before the start of the study. Therefore, the study, including the period of patient recruitment, lasted from September 2016 to August 2017. We have assumed such a time criterion to account for the timeliness and completeness of the patient data.

Based on individual medical records (source of the study), physicians personally completed questionnaires for enrolled patients concerning the main symptoms of SUDD, such as pain, abdominal tenderness, changes in bowel habits, and bloating, during the observation period. All variables were evaluated based on observations recorded in the patients' medical records and valued on a 4-point scale at the beginning of and during treatment.

The effectiveness of the treatment was assessed every 2 months. The survey also included a detailed history of the patients' DD history, the diagnostic methods used during the course of the disease, and the general results.

Hard copies of all the questionnaires, which were signed by the doctors and contained no patient data, were collected and stored at the research office. An electronic database was created after all the surveys had been obtained.

\section{Participants}

The study population consisted of male and female patients who were diagnosed with SUDD at least 6 months before the baseline visit and who had at least one episode of relapse. Patients were treated with six cycles of rifaximin. 
Symptomatic uncomplicated diverticular disease was defined as recurrent or persistent symptoms such as abdominal pain located mainly in the left lower quadrant, abdominal tenderness, bloating, constipation, diarrhoea, or alternating bowel habits in patients with previous endoscopically or radiologically diagnosed diverticula. Patients were eligible for treatment if they had no serious complications, such as peritonitis, abscess, fistula, stenosis, (sub)ileus, or bleeding.

Patients with ongoing $A D$, with inflammatory bowel disease (including microscopic colitis), or who were treated with systemic antibiotics (for reasons other than SUDD) were excluded from the study. Slight elevations of white blood cell count (WBC), erythrocyte sedimentation rate (ESR), C-reactive protein (CRP), or calprotectin in the absence of systemic symptoms such as fever were not exclusion criteria. Rifaximin treatment was defined as $800 \mathrm{mg}$ daily (two $200 \mathrm{mg}$ tablets twice a day) administered 7 days a month for 6 months.

All consecutive patients fulfilling the inclusion criteria during the study period were selected by the physicians on the basis of their medical records. In the end, only those patients who were actually treated with rifaximin, who were available for medical evaluation (vis it to a doctor's office) every 2 months, and for whom detailed documentation was available were evaluated.

\section{Variables}

Symptoms: abdominal pain, tenderness, changes in bowel habits, and bloating were assessed on a 4-point scale ( 0 - no symptoms, 1 - mild, 2 - moderate, 3 - severe) at each visit (at baseline and at 2, 4, and 6 months after the start of rifaximin treatment). The total symptom score was calculated by summing up the individual symptoms at each time point (maximal value: 18) and determining the median symptom severity (maximal value: 3 ).

Concomitant treatment of SUDD and other diseases as well as the recommended diet, which are also considered potential confounders or effect modifiers, have been identified as open issues. If laboratory tests were performed, the patients' WBC, ESR, CRP, and calprotectin values were obtained.

Age, gender, duration of the disease, number of flares, severity of symptoms at the beginning of treatment, number and type of drugs used, and changes in the severity of the symptoms were assessed as potential predictors of the disease course and responses to treatment.

\section{Data sources/measurement}

In Poland, electronic medical records are widely used. Therefore, patient data are fully accessible to all physicians who care for the patient, and they are objective with regard to patient information. To maximise the possible comparability of the assessment methods, we used the simplest scale (no symptoms and better, same or worse compared to the last visit: 0-3 points).

\section{Bias}

Because of the retrospective nature of the study, all bias could not be excluded. To best control for potential bias, we planned to include all consecutive patients treated with the study drug and minimise the exclusion criteria in an attempt to describe all the data that may affect the results. We also decided on a restrictive time criterion to limit the lengthy recruitment process, which may result in the loss of data. To avoid a positive bias, we analysed all the questionnaires received, although some of them did not precisely correspond to the survey protocol.

\section{Study size}

According to the Polish National Health Fund and Ministry of Health database, the prevalence of symptomatic DD in 2016 was over 543 per 100,000 people. This means that in Poland, there are approximately 206,000 people with symptomatic DD (based on the estimated population size).

The sample size was calculated with a confidence level of $95 \%$ and a margin error of $5 \%$ and was assumed to be 384 patients. Almost half a million outpatients visit gastroenterological and surgical clinics at last three times per year. Thus, we assumed that a 6-month study period would be sufficient to achieve our goal. Finally, we enrolled 300 patients, and a confidence level of $95 \%$ and a margin error of $5.6 \%$ were sufficient to perform the analysis.

\section{Statistical methods}

The baseline analysis of the endpoint and other efficacy assessments were based on the per protocol (PP) population, which included all patients treated with appropriate doses of rifaximin. However, key performance measures were also analysed to the intention to treat (ITT) population, defined as all enrolled in the study. We used the $\chi^{2}$ test, Fisher's exact test, or the Wilcoxon-Mann-Whitney test for statistical evaluation. With regard to differences in treatment, 95\% confidence intervals (Cls) were applied. All statistical tests were conducted at the bilateral nominal level error rate of 0.05 . The last observation carried forward method (LOCF) was used for calculation for early terminating patients. 


\section{Results}

\section{Participants}

A total of 300 surveys were received and analysed as ITT population. Among them, 294 had at least one diagnostic examination confirming DD. In the study group, 267 patients were treated with an appropriate dose of rifaximin and were included in further evaluation as a PP population (89\%). Five of the patients were evaluated after 4 months (Figure 1).

\section{Descriptive data}

The detailed characteristics of the participants are presented in Table I. The population had a median age of 65 years and was predominantly female. The mean duration of DD was 4.5 years, with a median of 3.3 years. The most common diagnostic method revealing diverticulosis was colonoscopy. A total of $61 \%$ of patients underwent more than one diagnostic procedure.

In addition, 88\% (60\% women) had at least one episode of relapse, and $19 \%$ (16\% women) had more than five SUDD episodes. At baseline, $91 \%$ of patients in whom laboratory tests were performed (224 patients) had at least one parameter (WBC, OB, CRP, calprotectin) elevated.

A total of $90 \%$ of patients before starting rifaximin therapy and $82 \%$ of patients at the beginning of treatment received other drugs (5-aminosalicylates (5-ASA),

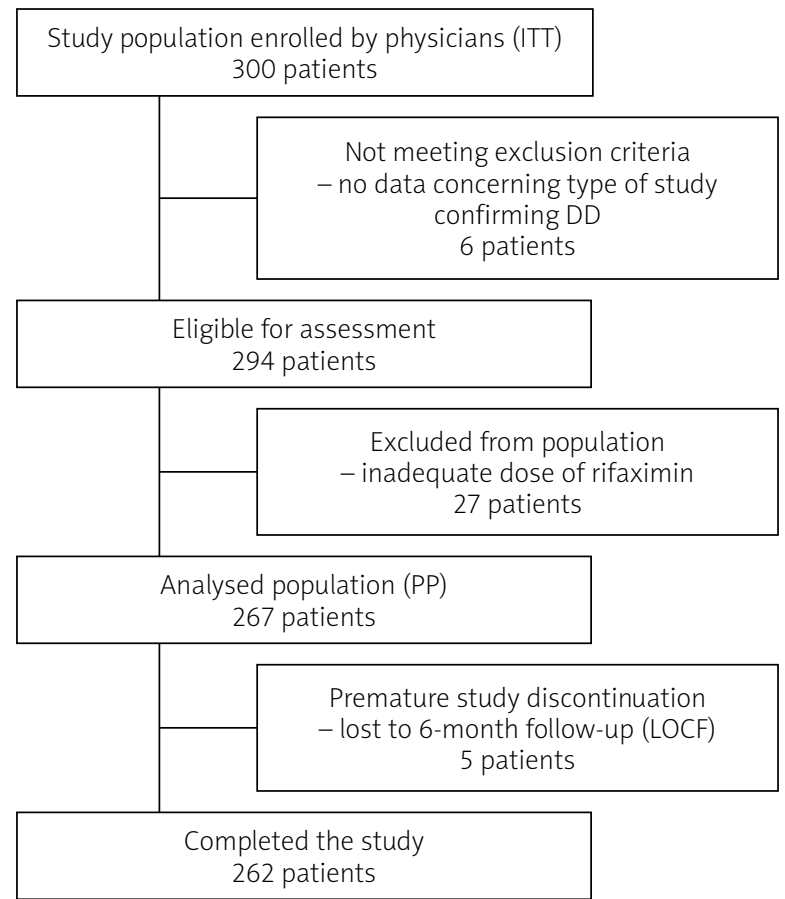

Figure 1. Patient disposition

ITT - intention to treat; $D D$ - diverticular disease, $P P$ - per protocol, LOCF-last observation carried forward. antibiotics, spasmolytics, prokinetics, probiotics, laxatives, and analgesics) with unproven efficacy to treat $\mathrm{DD}$, but this drug use was recorded as potentially relevant for further evaluation.

Table I. Patient demographics and baseline characteristics

\begin{tabular}{|c|c|}
\hline Variable & PP analysis \\
\hline Age [years] & $64.5(26-87)$ \\
\hline Male gender, $n(\%)$ & $87(33)$ \\
\hline Duration of DD [years] & $4.5(1-20)$ \\
\hline Number of flares & $4(0-50)$ \\
\hline Diagnostic test revealing DD (\%): & 100 \\
\hline Colonoscopy, $n(\%)$ & $250(93.7)$ \\
\hline Computed tomography, $n$ (\%) & $147(55)$ \\
\hline Abdominal ultrasound, $n(\%)$ & $87(32.6)$ \\
\hline Magnetic resonance, $n(\%)$ & $8(3)$ \\
\hline Baseline concomitant medication, $n(\%)$ : & $240(89.9)$ \\
\hline 5-aminosalicylates & $62(23.2)$ \\
\hline Antibiotics & $117(43.8)$ \\
\hline Fibre supplements & $19(6.7)$ \\
\hline Probiotics & $43(16.1)$ \\
\hline Prokinetics & $52(19.5)$ \\
\hline Spasmolytics & $126(47.2)$ \\
\hline NSAIDs & $9(3.4)$ \\
\hline Other analgesics & $24(9.4)$ \\
\hline Symptoms score at baseline (combine) (0-3 scale): & 1.8 \\
\hline Pain & 2.4 \\
\hline Tenderness & 2.1 \\
\hline Diarrhoea & 1.0 \\
\hline Constipation & 1.4 \\
\hline Changing bowel habits & 0.9 \\
\hline Bloating & 2.4 \\
\hline $\begin{array}{l}\text { If performed, baseline laboratory test elevated } \\
\text { (combine), } n(\%) \text { : }\end{array}$ & $203(91)$ \\
\hline WBC & $169(71)$ \\
\hline CRP & $201(92)$ \\
\hline ESR & $76(61)$ \\
\hline Number of patients assessed & 267 \\
\hline
\end{tabular}


A high-fibre diet was recommended in $70 \%$ of patients. Because the fibre supplements have high heterogeneity regarding the various forms of fibre (soluble, insoluble, viscous, non-viscous), we were unable to conduct a thorough analysis of the ordered supplementation of fibre. The total average symptom score at the baseline level was $1.8(0-3)$. The most severe symptoms were pain and bloating. All patients (except five) were followed for 6 consecutive months.

\section{Main results and other analyses}

After 6 months of treatment with rifaximin, we found a statistically significant reduction in all symptoms, regarding both the total symptom score and each symptom. All combined symptoms decreased from a mean intensity of 1.8 to 0.2 (max. 3 points; $p<0.001$ ) and the total symptom score from 9.37 (max. 18 points)

\section{A}

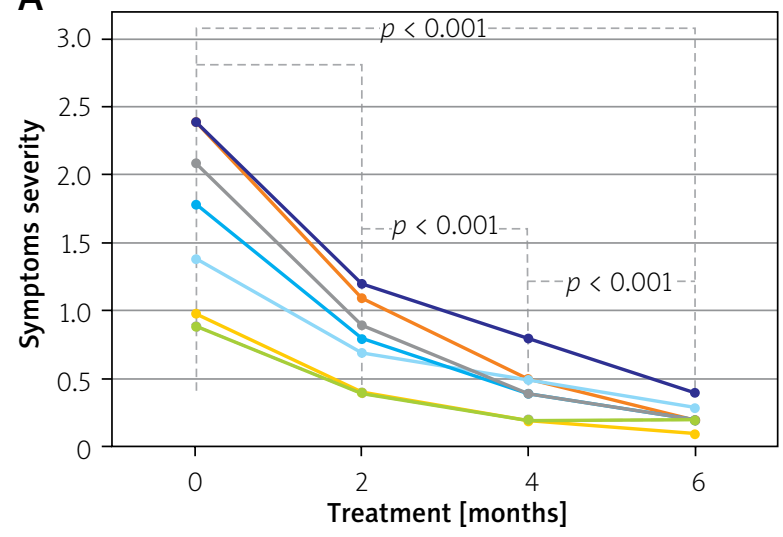

$\rightarrow$ All symptoms $\rightarrow$ Pain $\rightarrow$ Tenderness

$\because$ Alteration $\rightarrow$ Bloating to 1.35 ( $p<0.0001)$. Abdominal pain decreased from 2.4 to 0.2 points; tenderness from 2.1 to 0.2 ; diarrhoea from 1.0 to 0.1 ; constipation from 1.4 to 0.3 ; altered bowel habits from 0.9 to 0.2 , and bloating from 2.4 to 0.4. No differences were found between the ITT and PP analyses, and the $p$-value was $<0.001$ for all evaluations (detailed analyses - Figures 2 A, B). A total of $3.4 \%$ of patients were asymptomatic (resolution of all symptoms with 0 points indicated on the scale) after 2 months of treatment, $13.5 \%$ after 4 months and $37.5 \%$ after 6 months ( $p$ for all values $<0.001$ ). The greatest symptom improvement after 6 treatment cycles was observed in diarrhoea, abdominal tenderness, and pain (79.7; 79.2 , and $76.9 \%$, respectively). Except for that of altered bowel habits after 2 months of treatment, a significant improvement was observed for each symptom after every 2 months of assessment $(p<0.001)$ (Table II).

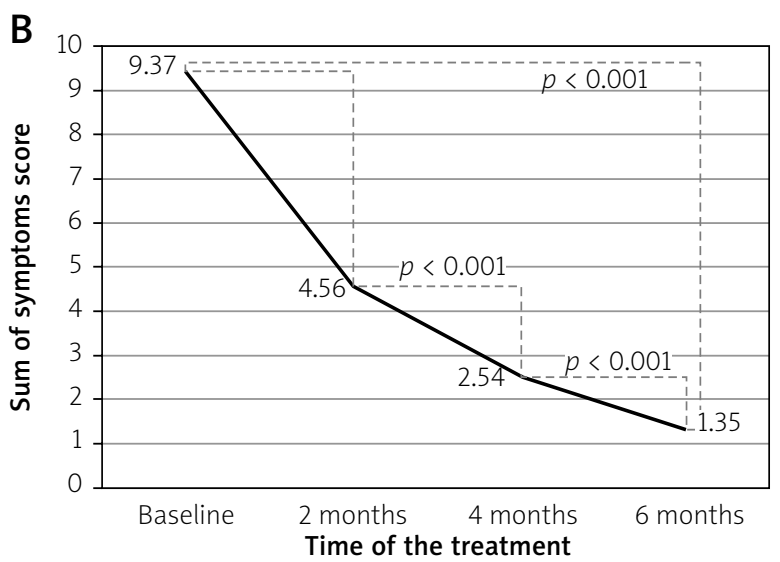

Figure 2. Detailed analysis of symptom reduction with statistical assessments. The $p$-value was calculated for each variable for each analysis every 2 months. A - Mean symptom severity (0-3 points scale). B - Mean symptom severity (sum of symptoms $0-18$ points scale)

Table II. Assessment of complete symptom resolution: answer 0 on a 4-point scale. For each symptom between assessments, it was a significant improvement (except alteration of bowel habits after 2 months - in bold)

\begin{tabular}{|c|c|c|c|c|}
\hline Parameter & $\begin{array}{l}\text { All patients with } \\
\text { symptom }(n)\end{array}$ & $\begin{array}{c}\text { "0" after } 2 \text { months } \\
n(\%)\end{array}$ & $\begin{array}{c}\text { “0" after } 4 \text { months } \\
n(\%)\end{array}$ & $\begin{array}{c}\text { "0" after } 6 \text { months } \\
n(\%)\end{array}$ \\
\hline Pain & 260 & $39(15)$ & $121(46.5)$ & $200(76.9)$ \\
\hline Tenderness & 251 & $56(22.3)$ & $134(53.4)$ & $200(79.7)$ \\
\hline Diarrhoea & 120 & $45(37.5)$ & $69(57.5)$ & $95(79.2)$ \\
\hline Constipation & 148 & 35 (23.6) & 59 (39.9) & $85(57.4)$ \\
\hline Alteration & 99 & $34(33.4)$ & $43(43.4)$ & $65(65.6)$ \\
\hline Bloating & 257 & $32(12.4)$ & $77(30)$ & $145(56.4)$ \\
\hline All symptoms assessed & 267 & $9(3.4)$ & $36(13.5)$ & $100(37.4)$ \\
\hline
\end{tabular}




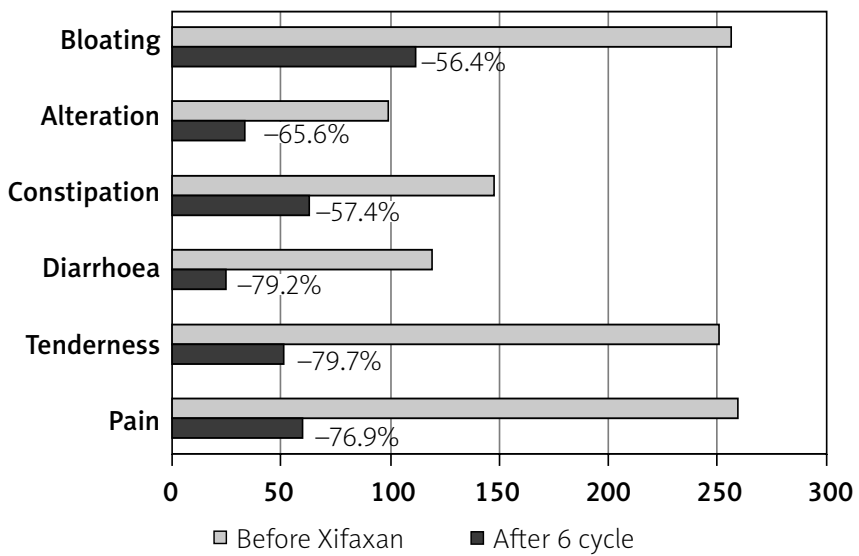

Figure 3. Number of patients with complete symptom resolution after 6 months compared with start of treatment

A large proportion of patients were symptom free after 6 months of treatment (Figure 3). We found significant normalisation of all analysed laboratory tests. Leucocytosis, ESR, and CRP were decreased in $80 \%, 84 \%$, and $46.8 \%$ of patients, respectively (Figure 4 ). There was

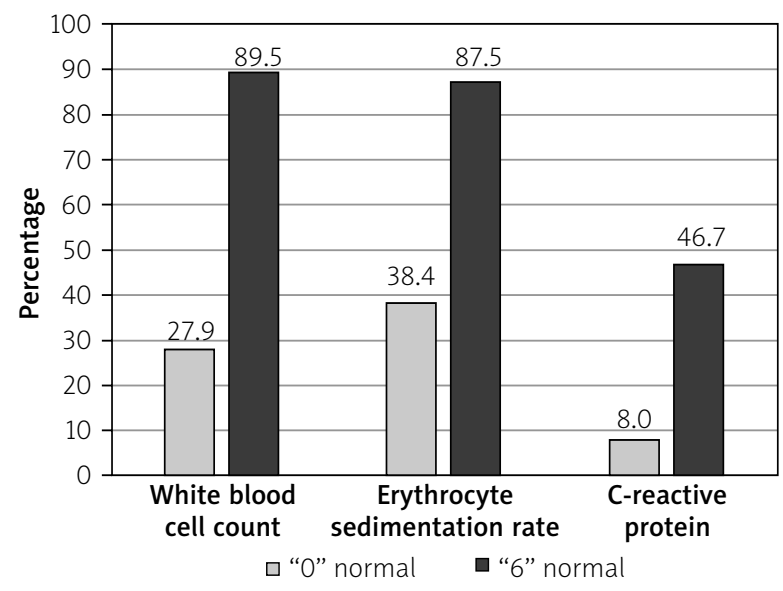

Figure 4. Laboratory test normalisation during rifaximin treatment. " 0 " - start of rifaximin treatment, " 6 " - after 6 months of rifaximin treatment. Values are presented as \% no statistically significant correlation between the severity of symptoms at baseline and the reduction in laboratory abnormalities. In the multivariate analysis, we did not find any differences between genders regarding duration of the disease, number of flares, and severity of symptoms at baseline and after treatment. We also examined whether other potential cofounders are risk factors, but we observed that duration of the disease, severity of symptoms, and number of flares were not risk factors that influenced treatment effectiveness in our cohort. The mean age (55-64 years) was associated with a more severe disease course (Tables III-V).

We also analysed concomitant treatment. We found that during treatment with the rifaximin regimen, there was a significant reduction in the number and types of other drugs prescribed (Table VI). Only the decision to introduce rifaximin significantly decreased the use of any drug evaluated, including systemic antibiotics, spasmolytics, and analgesics $(p<0.05)$. The largest reduction (93.2\%) was observed for prescribing systemic antibiotics. After 2 months of treatment the use of analgesics and spasmolytics decreased significantly ( $p<$ $0.05)$, and after 6 months any drug evaluated as well as 5-ASA showed significant decreases $(p<0.05)$. No

Table III. Age (years)-adjusted estimates. Sum of symptoms score (maximum value 18 points); $p<0.05$ indicates statistical significance

\begin{tabular}{|c|c|c|c|c|c|c|}
\hline Age [years] & $\begin{array}{l}\text { Number of } \\
\text { patients }\end{array}$ & Male & $\begin{array}{c}\text { Duration of the } \\
\text { disease }\end{array}$ & No flares & $\begin{array}{l}\text { Symptoms } \\
\text { baseline }\end{array}$ & $\begin{array}{c}\text { Symptoms after } \\
\text { treatment }\end{array}$ \\
\hline$<45$ & 13 & 8 & 4.5 & 4 & 9.69 & 0.76 \\
\hline $45-54$ & 26 & 10 & 4.5 & 4 & 9.65 & 1.3 \\
\hline $55-64$ & 86 & 25 & 4.5 & 4 & 10.02 & 1.46 \\
\hline $65-74$ & 99 & 30 & 4.5 & 4 & 9.75 & 1.43 \\
\hline $75-84$ & 37 & 12 & 4.6 & 4 & 8.9 & 1.03 \\
\hline$>84$ & 6 & 2 & 5.5 & 4.7 & 9.85 & 0.57 \\
\hline
\end{tabular}


Table IV. Disease duration (years)-adjusted estimates. Sum of symptoms score (maximum value 18 points); $p=0.4$ (n.s.)

\begin{tabular}{lcccc} 
Duration of the disease & Number of patients & Number of flares & Symptoms baseline & Symptoms after treatment \\
\hline $0-4$ & 161 & 4 & 9.89 & 1.15 \\
\hline $5-9$ & 78 & 6.3 & 9.43 & 1.58 \\
\hline $10-14$ & 19 & 9 & 9.75 & 1.43 \\
\hline$>14$ & 12 & 9 & 9.5 & 1.83
\end{tabular}

effects on prokinetics use were found. During the observation period, there was no need to reintroduce any of the previously discontinued medicines.

A total of $31 \%$ of patients adhered to the recommended high-fibre diet, and no differences were found between patients administered rifaximin and those who were using 5-ASA or consuming a high-fibre diet, with reference to the severity and reduction of their symptoms.

\section{Discussion}

Key results

In this first 6-month retrospective observational survey study of rifaximin alone for SUDD, prolonged cyclic rifaximin treatment was effective; it continuously reduced all symptoms and prevented the recurrence of exacerbation irrespective of other treatments applied. Analyses consistently showed a significant reduction in each clinical and laboratory parameter assessed during each visit. A large proportion of patients were completely asymptomatic after 6 months of treatment. In our cohort, we did not find any potential predictors, cofounders, or effect modifiers regarding age, gender, duration of the disease, number of flares, or prescribed
Table V. Number of flares-adjusted estimates. Sum of symptoms score (maximum value 18 points); $p=0.34$ (n.s.)

\begin{tabular}{lccc}
$\begin{array}{l}\text { Number } \\
\text { of flares }\end{array}$ & $\begin{array}{c}\text { Number } \\
\text { of patients }\end{array}$ & $\begin{array}{c}\text { Symptoms } \\
\text { baseline }\end{array}$ & $\begin{array}{c}\text { Symptoms } \\
\text { after treatment }\end{array}$ \\
\hline $0-4$ & 199 & 10.05 & 1.27 \\
\hline $5-9$ & 45 & 9.04 & 1.62 \\
\hline $10-14$ & 13 & 8.46 & 1.23 \\
\hline$>14$ & 10 & 8.2 & 1.2 \\
\hline
\end{tabular}

drugs. During rifaximin treatment, the number of other drugs used, such as systemic antibiotics, 5-ASA, and spasmolytics, decreased significantly. No differences in rifaximin efficacy were found in patients additionally treated with a high-fibre diet or 5-ASA.

\section{Limitations}

Our study has some limitations that should be taken into account. Due to its retrospective nature, we were unable to adequately recruit a sufficient study group, so

Table VI. Changes in the concomitant treatment used after introducing rifaximin

\begin{tabular}{|c|c|c|c|c|c|}
\hline Concomitant medication & Before rifaximin & Baseline & 2 months & 4 months & 6 months \\
\hline Combined, $n(\%)$ & $235(88)$ & $131(49.1)$ & $125(46.8)$ & $111(41.6)$ & $97(36.3)$ \\
\hline 5-aminosalicylates, $n(\%)$ & $62(23.2)$ & $47(17.6)$ & $42(15.7)$ & $33(12.3)$ & $27(10.1)$ \\
\hline Antibiotics, $n(\%)$ & $117(43.8)$ & $8(3)$ & $5(1.9)$ & $3(1.1)$ & $3(1.1)$ \\
\hline High-fibre diet, $n(\%)$ & $174^{\star}(65)$ & $82^{* *}(30.7)$ & $68^{* *}(25.5)$ & $70^{\star *}(26.2)$ & $75^{* *}(28.1)$ \\
\hline Probiotics, $n(\%)$ & $43(16.1)$ & $61(22.8)$ & $55(20.6)$ & 47 (17.6) & $46(17.2)$ \\
\hline Prokinetics, $n$ (\%) & $52(19.5)$ & $44(16.5)$ & $47(17.6)$ & $36(13.5)$ & $38(14.2)$ \\
\hline Spasmolytics, $n(\%)$ & $126(47.2)$ & $103(38.6)$ & $79(29.6)$ & $56(21)$ & $40(15)$ \\
\hline NSAIDs, $n(\%)$ & $9(3.4)$ & $2(0.75)$ & $2(0.75)$ & $1(0.37)$ & $0(0)$ \\
\hline Other analgesics, $n(\%)$ & $24(9.4)$ & $14(5.2)$ & $0(0)$ & $0(0)$ & $0(0)$ \\
\hline
\end{tabular}

Before rifaximin - earlier treatment before decision of introduction of rifaximin. Baseline-onset of rifaximin treatment. *Diet prescribed by the doctor; **patients following recommendation, NSAIDs - nonsteroidal anti-inflammatory drugs. 
the cohort was slightly smaller than expected. Nevertheless, we reached statistical power to perform the study.

Of course, it should be noted that the doctors chose the patients to include. We are therefore concerned that, despite the restrictive inclusion criteria, the doctors selected only those who responded exceptionally well to the treatment. This argument is the most difficult to address; however, it should be considered that due to the high cost of rifaximin treatment, the group of patients taking this medication is not large, and the efficacy of the drug is carefully analysed in each patient. Therefore, if the patient did not respond to treatment after the first cycle, treatment was likely to be discontinued, and these patients were not included in our study. We were not able to estimate how many patients did not respond to the initial treatment. The aim of the study, however, was not only to assess the response to treatment but also to assess the need for cyclic therapy in people who responded to the initial treatment.

The last potential bias that should be discussed is a correct diagnosis. Although it may be difficult to diagnose DD solely on the basis of symptoms, we believe that the current diagnostic criteria are so precise that there should be no errors in the interpretation of symptoms, especially considering that only patients with confirmed presence of diverticula on imaging or via endoscopic examinations were included in further analyses.

\section{Interpretation}

Very few studies, each with heterogeneous inclusion criteria and different endpoints, investigated the impact of prolonged treatment with rifaximin alone and in combination with other drugs on the course of DD. Earlier, four large-scale studies by Papi et al. [17, 18], Latella et al. [19], and Colecchia et al. [20], which were later confirmed by the metanalysis of Bianchi et al. [21], assessed the efficacy of rifaximin but only in combination with fibre supplements and with restrictive criteria that excluded other treatments. There were no interim analyses in these studies; thus, we cannot directly compare the results. However, considering these limitations, our data are comparable, especially when considering improvements in laboratory testing. The efficacy of rifaximin in preventing the occurrence and recurrence of diverticulitis has been discussed in several studies [22-24]. Even if rifaximin was proven effective, these reports are not directly comparable. However, a study by Banasiewicz et al. found that 6 and 12 treatment cycles of rifaximin not only prevents diverticulitis but also improves the quality of life. Patient response to rifaximin can be adapted as a surrogate marker for SUDD remission and is comparable to our results.
At the time of our study, only two other publications assessing rifaximin monotherapy were available [14, 15]. The study groups in both reports were different, and they were conducted over a period of 3 months. For this reason, we can only compare a subset of the results. Stallinger et al. proved that every cycle of rifaximin improves SUDD symptoms, which is consistent with our finding, but in their cohort statistical significance was not achieved until the third month of treatment. It is particularly difficult to interpret these results, especially since their cohort was the largest one (over 1000 participants). The differences can be partially explained by the variance in the inclusion criteria (in Stallinger's study, patients had to experience a minimum of three episodes of SUDD exacerbation in the preceding year) and partially by differences in the local microbiota compositions in Austria and Poland. In our study, patients reported significant relief after the first cycle, but the improvement from cycle to cycle also reached statistical significance. This is comparable to the results observed in the Moniuszko and Rydzewska study [15]. Their study was slightly different because they recruited patients experiencing their first episodes of various forms of DD. For this reason, their population had a better response to initial treatment. Their population was also slightly smaller and comprised 142 patients, which may explain why there were no potential confounders, risk factors, and subgroups identified in their study. As with our findings, they also found substantial reduction in other drugs used (but they did not assess the statistical significance).

Last, but not least, is the influence of a high-fibre diet. Research on this topic is inconsistent, perhaps because of different diet regimens and types of supplements used [25]. In a study published in 2014, Crowe et al. found that only soluble fibre is relevant in the prevention of SUDD and diverticulitis [26]. In our cohort, there was no possibility to differentiate between the types of dietary fibres used; therefore, no differences were found between groups consuming a high-fibre diet and those consuming a standard diet. Another important issue was patient compliance. We found that less than half of the patients followed the recommended diet (47.2\%). Therefore, the best source of fibre supplementation for the Polish population should still be determined (probably supplements due to expected better compliance).

Our study has an advantage: to the best of our knowledge, this is the first longitudinal real-life study evaluating the use of rifaximin with different drugs and showing a gradual improvement in symptoms, regardless of other treatments, or even a reduction in the use of other therapies (from $68 \%$ to $59 \%, p<0.01$ ) in favour 
of rifaximin. We were also able to identify the minimum treatment time needed to achieve remission in SUDD; this duration was estimated at 6 months in patients with recurrent disease.

\section{Generalisability}

In conclusion, our study shows that the cyclical rifaximin regimen is effective in inducing and maintaining remission in patients with SUDD, who responded to initial treatment but only when administered for six cycles. Taking into account existing evidence that SUDD is a chronic disease as well as the results from previous studies and our data, it seems reasonable to propose an algorithm for the management of various forms of DD and to specify the names of the different durations of treatment:

a) initial induction of remission - first cycle of rifaximin,

b) partial induction of remission -3 cycles of rifaximin,

c) complete induction of remission -6 cycles of rifaximin, and

d) maintenance of remission - 12 to 24 cycles of rifaximin (not yet assessed).

During the first episode of SUDD, where symptoms are the main problem and the chief goal is to reduce symptoms, the proposed treatment regimen is three cycles. In cases of SUDD symptom relapse, the main goal is to prevent future relapse. In those instances, the proposed treatment is six cycles. In post-diverticulitis SUDD, the most important goal is to induce deep remission because the main problem is the severity of symptoms. Therefore, the main objective should be to prevent complications; to this end, we propose 12 to 24 cycles of rifaximin therapy.

\section{Acknowledgments}

All participants' data (surveys) as well as all electronic databases and statistical calculation are accessible in study centre in the Centre of Oncology, Warsaw, Poland.

\section{Conflict of interest}

Anna Pietrzak, Tomasz Banasiewicz, Adam Dziki and Jaroslaw Regula were remunerated by Alfasigma's lecturers during the last two years.

\section{References}

1. Everhart JE, Ruhl CE. Burden of digestive diseases in the United States part II: lower gastrointestinal diseases. Gastroenterology 2009; 136: 741-54.

2. Delvaux M. Diverticular disease of the colon in Europe: epidemiology, impact on citizen health and prevention. Aliment Pharmacol Ther 2003; 18 Suppl 3: 71-4.

3. Peery AF, Keku TO, Martin CF, et al. Distribution and characteristics of colonic diverticula in a United States screening population. Clin Gastroenterol Hepatol 2016; 14: 980-5. e981.

4. Petruzziello L, lacopini F, Bulajic M, et al. Uncomplicated diverticular disease of the colon. Aliment Pharmacol Ther 2006; 23: 1379-91.

5. Shahedi K, Fuller G, Bolus R, et al. Long-term risk of acute diverticulitis among patients with incidental diverticulosis found during colonoscopy. Clin Gastroenterol Hepatol 2013; 11: 1609-13.

6. Tursi A, Mario FD, Grillo S, et al. Natural history of symptomatic uncomplicated diverticular disease: a 13-year prospective study. Gastroenterology 2017; 152: S807.

7. Peery AF, Dellon ES, Lund J, et al. Burden of gastrointestinal disease in the United States: 2012 update. Gastroenterology 2012; 143: 1179-87.e1171-e1173.

8. Schieffer KM, Sabey K, Wright JR, et al. The microbial ecosystem distinguishes chronically diseased tissue from adjacent tissue in the sigmoid colon of chronic, recurrent diverticulitis patients. Sci Rep 2017; 7: 8467.

9. Tursi A, Mastromarino P, Capobianco D, et al. Assessment of fecal microbiota and fecal metabolome in symptomatic uncomplicated diverticular disease of the colon. J Clin Gastroenterol 2016; 50 Suppl 1: S9-12.

10. Barbara G, Scaioli E, Barbaro MR, et al. Gut microbiota, metabolome and immune signatures in patients with uncomplicated diverticular disease. Gut 2017; 66: 1252-61.

11. Carabotti M, Annibale B. Treatment of diverticular disease: an update on latest evidence and clinical implications. Drugs Context 2018; 7: 212526.

12. Ponziani FR, Zocco MA, D'Aversa F, et al. Eubiotic properties of rifaximin: disruption of the traditional concepts in gut microbiota modulation. World J Gastroenterol 2017; 23: 4491-9.

13. Stollman N, Smalley W, Hirano I. American Gastroenterological Association Institute guideline on the management of acute diverticulitis. Gastroenterology 2015; 149: 1944-9.

14. Stallinger S, Eller N, Hogenauer C. Non-interventional study evaluating efficacy and tolerability of rifaximin for treatment of uncomplicated diverticular disease. Wien Klin Wochenschr 2014; 126: 9-14.

15. Moniuszko A, Rydzewska G. The effect of cyclic rifaximin therapy on symptoms of diverticular disease from the perspective of the gastroenterology outpatient clinic: a "real-life" study. Gastroenterology Rev 2017; 12: 145-51.

16. von Elm E, Altman DG, Egger M, et al. The strengthening the reporting of observational studies in epidemiology (STROBE) statement: guidelines for reporting observational studies. Lancet 2007; 370: 1453-7.

17. Papi C, Ciaco A, Koch M, Capurso L. Efficacy of rifaximin on symptoms of uncomplicated diverticular disease of the colon. A pilot multicentre open trial. Diverticular disease study group. Ital J Gastroenterol 1992; 24: 452-6.

18. Papi C, Ciaco A, Koch M, Capurso L. Efficacy of rifaximin in the treatment of symptomatic diverticular disease of the colon. A multicentre double-blind placebo-controlled trial. Aliment Pharmacol Ther 1995; 9: 33-9.

19. Latella G, Pimpo MT, Sottili S, et al. Rifaximin improves symptoms of acquired uncomplicated diverticular disease of the colon. Int J Colorectal Dis 2003; 18: 55-62. 
20. Colecchia A, Vestito A, Pasqui F, et al. Efficacy of long term cyclic administration of the poorly absorbed antibiotic rifaximin in symptomatic, uncomplicated colonic diverticular disease. World J Gastroenterol 2007; 13: 264-9.

21. Bianchi M, Festa V, Moretti A, et al. Meta-analysis: long-term therapy with rifaximin in the management of uncomplicated diverticular disease. Aliment Pharmacol Ther 2011; 33: 902-10.

22. Banasiewicz T, Francuzik W, Bobkiewicz A, et al. The influence of rifaximin on diverticulitis rate and quality of life in patients with diverticulosis. Pol Przegl Chir 2017; 89: 22-31.

23. Lanas A, Ponce J, Bignamini A, Mearin F. One year intermittent rifaximin plus fibre supplementation vs. fibre supplementation alone to prevent diverticulitis recurrence: a proof-of-concept study. Dig Liver Dis 2013; 45: 104-9.

24. Festa V, Alegiani SS, Chiesara F, et al. Retrospective comparison of long-term ten-day/month rifaximin or mesalazine in prevention of relapse in acute diverticulitis. Eur Rev Med Pharmacol Sci 2017; 21: 1397-404.

25. Carabotti M, Annibale B, Severi C, Lahner E. Role of fiber in symptomatic uncomplicated diverticular disease: a systematic review. Nutrients 2017; 9: E161.

26. Crowe FL, Balkwill A, Cairns BJ, et al. Source of dietary fibre and diverticular disease incidence: a prospective study of UK women. Gut 2014; 63: 1450-6.

Received: 20.11.2018

Accepted: 4.01.2019 